TITLE:

\title{
MALDI-TOF/MS analyses of decomposition behavior of beech xylan as treated by semi-flow hot- compressed water
}

\section{$\operatorname{AUTHOR}(S)$ :}

Nakahara, Yu; Yamauchi, Kazuchika; Saka, Shiro

\section{CITATION:}

Nakahara, Yu ...[et al]. MALDI-TOF/MS analyses of decomposition behavior of beech xylan as treated by semi-flow hot-compressed water. Journal of Wood Science 2014, 60(3): 225 231

ISSUE DATE:

2014-03-15

URL:

http://hdl.handle.net/2433/198673

\section{RIGHT:}

The final publication is available at Springer via http://dx.doi.org/10.1007/s10086-0141396-0.; This is not the published version. Please cite only the published version.; この論 文は出版社版でありません。引用の際には出版社版をご確認ご利用ください。 


\section{Title}

MALDI-TOF/MS analyses of decomposition behavior of beech xylan as treated by semi-flow hot-compressed water

\section{The type of artile}

Original article

\section{The authors' full names, affiliations, and addresses}

Yu Nakahara · Kazuchika Yamauchi · Shiro Saka

Department of Socio-environmental Energy Science, Graduate School of Energy Science, Kyoto University, Kyoto, Japan,

Yoshida-honmachi, Sakyo-ku, Kyoto 606-8501, Japan

Tel. +81-75-753-4738; Fax: +81-75-753-4738

e-mail: saka@energy.kyoto-u.ac.jp

\section{Key words}

Japanese beech $\cdot$ Hot-compressed water $O$-acetyl-4- $O$-methylgulucuronoxylan $\cdot$ MALDI-TOF/MS . Native xylan 


\section{Abstract}

Japanese beech (Fagus crenata) was treated with semi-flow hot-compressed water at various temperatures of $150-230{ }^{\circ} \mathrm{C}$ under $10 \mathrm{MPa}$. The obtained various products were, then, analyzed with matrix-assisted laser desorption/ionization time of flight mass spectrometry (MALDI-TOF/MS). In a temperature range of $150{ }^{\circ} \mathrm{C}$ up to $210{ }^{\circ} \mathrm{C}$, however, no hydrolyzed products were found, and at $210{ }^{\circ} \mathrm{C} / 10 \mathrm{MPa}, \quad O$-acetyl-4- $O$-methylglucuronoxylo-oligosaccharides $\quad\left(\mathrm{X}_{n} \mathrm{Ac}_{m} \mathrm{MG}_{i}\right)$ and $O$-acetyl-xylo-oligosaccharides $\left(\mathrm{X}_{n} \mathrm{Ac}_{m}\right)$ were obtained, indicating the first cleavage of native xylan $\beta$-1,4-glycosidic linkages followed by a cleavage of $\alpha$-1,2-glycosidic linkage in 4- $O$-methylglucuronic acid (MG) residue at mainly $220-230{ }^{\circ} \mathrm{C}$ under $10 \mathrm{MPa}$. At subsequent stage of $230{ }^{\circ} \mathrm{C} / 10 \mathrm{MPa}, \mathrm{X}_{n} \mathrm{Ac}_{m}$ were predominantly recovered. As the treatment was prolonged at $230{ }^{\circ} \mathrm{C}$, $\mathrm{X}_{n} \mathrm{Ac}_{m}$ were reduced, but remained to some extent, indicating that the acetyl group which is hydrolyzed to be acetic acid is more resistant than MG residue. In such a stage of treatment, cellulose started to hydrolyze to be cello-oligosaccharides. These lines of evidence can clearly indicate the hydrolysis pathway of native $O$-acetyl-4- $O$-methylglucuronoxylan as treated by hot-compressed water. Thus, xylo-oligosaccharides recovered in a very early stage of the semi-flow hot-compressed water treatment preserve native $O$-acetyl-4- $O$-methylglucuronoxylan. 


\section{Introduction}

The major cell wall components of wood are composed of cellulose, hemicelluloses and lignin, in which cellulose is first synthesized as crystalline cellulose microfibrils and then associated with hemicelluloses. Such carbohydrates are then encrusted with lignin to form lignocellulosic cell wall microstructure [1]. Lignin is a hydrophobic aromatic polymer, while cellulose is not only hydrophobic due to its crystalline nature by numerous hydrogen bonds but also hydrophilic because of its numerous hydroxyl groups over the surface of cellulose microfibrils. Although it has been expected that hemicelluloses mediate between these alien substances, their functions have not been elucidated in detail. Thus, it is of importance to know about the structure of the native hemicellulose.

Since 1957 [2], Timell and co-workers have intensively investigated and speculated the chemical composition and the structure of the hemicelluloses in different wood species $[3,4]$. In one of their studies, white birch (Betura papyrifera) was found to contain one 4- $O$-methylglucuronic acid residue and 3.6 acetyl groups per 10 xylose units. In addition, the number- and weight-average degrees of polymerization were, respectively, determined as 180 and 470 by using viscosity, osmotic pressure, and light-scattering measurements [5]. Furthermore, paper chromatography was carried out for determining sugars derived from the xylan, followed by qualitative and quantitative analyses of them. The obtained results showed the average properties of the native xylans such as number of each residue per one xylose unit.

Since a series of the studies by Timell and co-workers, Rosell and Svensson reported that the 
4-O-methylglucuronic acid residues are irregularly distributed in birch (Betura verrucosa) xylan extracted with alkali, based on the results of analyses with gas-chromatography mass-spectrometry (GC-MS), high performance liquid chromatography (HPLC), infrared absorption spectrometry and optical rotation measurement [6]. However, in general, acetyl group is readily removed by means of extraction with alkali solutions, the migration of which has been found to occur by Caregg [7]. Thus, Reicher et al. investigated the condition that acetyl group in bracatinga (Mimosa scabrella) does not migrate [8], where highly uneven distribution of acetyl groups in the xylan was determined in analyses with ${ }^{13} \mathrm{C}$ nuclear magnetic resonance (NMR) and fast atom bombardment mass spectrometry (FAB-MS) [9]. These studies $[8,9]$ are, however, based on rather complicated method for extraction with a low yield, the results of which might not be able to reflect the whole native structure of xylan.

In recent years, hot-compressed water treatment has been noted as the efficient method for hydrolysis of lignocellulosics including woody biomass $[10,11]$. This method uses no catalysts such as acid or alkali, but water. Under the conditions by the two-step hot-compressed water treatment at $230{ }^{\circ} \mathrm{C} / 10 \mathrm{MPa}$ and $270{ }^{\circ} \mathrm{C} / 10 \mathrm{MPa}$ for $15 \mathrm{~min}$ each applied, polysaccharides and lignin were found to be decomposed rather selectively and efficiently $[10,11]$, where water acts as reagent, solvent and catalyst [12]. As a result, a major hardwood hemicellulose in Japanese beech, $O$-acetyl-4- $O$-methylglucuronoxylan, was decomposed into xylose, xylo-oligosaccharides, glucuronic acid, and acetic acid [10]. However, the detailed hydrolysis pathway remains unclear 
[10].

In this study, therefore, matrix-assisted laser desorption/ionization time of flight mass spectrometry (MALDI-TOF/MS), which enables non-destructive structural analysis without denaturation of the molecular structure, was carried out to discuss the detailed hydrolysis pathway that the xylo-oligosaccharides were recovered, so as to elucidate the native structure of $O$-acetyl-4- $O$-methylglucuronoxylan. 


\section{Materials and Methods}

Materials and chemicals

Wood flour of Japanese beech (Fagus crenata) passing through an 18-mesh screen was prepared for hot-compressed water treatment. The obtained wood flour was, then, extracted with acetone for $8 \mathrm{~h}$ and dried at $45{ }^{\circ} \mathrm{C}$ for $24 \mathrm{~h}$. One $\mathrm{g}$ of the dried flour was used for subsequent semi-flow hot-compressed water treatment [10]. Chemicals used in this study were all of reagent grade without purification, unless otherwise mentioned.

Semi-flow hot-compressed water treatment

The semi-flow hot-compressed water treatment system and operational procedure as described elsewhere [10] were used. Under $10 \mathrm{MPa}$ in pressure and the flow-rate of $10.0 \mathrm{ml} / \mathrm{min}$, treatment temperature was started from $150{ }^{\circ} \mathrm{C}$, risen up to $230{ }^{\circ} \mathrm{C}$ and remained constant at $230{ }^{\circ} \mathrm{C}$; during this treatment, hemicelluloses were hydrolyzed as referred in the previous report [10]. The semi-flow hot-compressed water-soluble portion was, then, collected every $1 \mathrm{~min}$ and analyzed as shown below.

Analytical methods 
The aliquot of $1 \mu \mathrm{l}$ of each fraction of water-soluble portion collected were firstly pipetted on a stainless steel sample slide for MALDI-TOF/MS analysis. The 2,5-dihydroxybenzoic acid (DHB; MALDI-MS grade, SIGMA, U.S.A.) was used as the matrix for oligosaccharides analyses [13, 14]. DHB was then treated to prepare $10 \mathrm{mg} / \mathrm{ml}$ matrix solution of a mixture of $50 \%$ acetonitrile (NACALAI-TESQUE INC., Japan) and $0.5 \%$ trifluoroacetic acid (TFA; FLUKA, U.S.A.) aqueous solution (v/v). In the same way, $10 \mathrm{mg} / \mathrm{ml}$ sodium trifluoroacetate (TFANa; Wako Pure Chemical Industries, Ltd., Japan) solution was also prepared to be mixed into the samples for MALDI-TOF/MS analyses; oligosaccharides are known to provide the alkali metals cationized species such as [M + $\mathrm{Na}]^{+},[\mathrm{M}+\mathrm{K}]^{+}[13]$. To simplify the analyses of the obtained spectra, TFANa was added into the samples for convergence to $[\mathrm{M}+\mathrm{Na}]^{+}$. The aliquot of $1 \mu \mathrm{l}$ of the matrix solution and $0.5 \mu \mathrm{l}$ of the TFANa solution were added into the water soluble portion on a spot in the sample slide and dried under an ambient temperature and pressure condition.

As standards of oligosaccharides, xylo-tetraose $\left(\mathrm{X}_{4}\right)$, xylo-pentaose $\left(\mathrm{X}_{5}\right)$ and xylo-hexaose $\left(\mathrm{X}_{6}\right)$ (Megazyme International Ireland, Ireland) were used, the solutions of which were, respectively, prepared to be $10 \mathrm{mg} / \mathrm{ml}$ of $50 \%$ acetonitrile and $0.5 \%$ trifluoroacetic acid TFANa aqueous solutions (v/v) as well as the matrix solution. All the oligosaccharide solutions equal in volume were mixed, then $1 \mu \mathrm{l}$ of the mixture was further mixed with $1 \mu \mathrm{l}$ of the matrix solution and $0.5 \mu \mathrm{l}$ of TFANa solution on the sample slide. 
Using this sample slide, the MALDI-TOF/MS spectra were acquired on an Axima-CRF instrument (Shimadzu Co., Japan), operating in the linear and positive ion mode with an accelerating potential of $20 \mathrm{kV}$ and equipped with a UV pulsed laser $\left(\mathrm{N}_{2}, \lambda=337 \mathrm{~nm}, 200 \mu \mathrm{J}\right.$ per shot $)$. The data were acquired by scanning the sample spot manually and averaging 200 laser shots [15]. 


\section{Results and Discussion}

MALDI-TOF/MS analyses of the oligosaccharide standards

Fig. 1 shows MALDI-TOF/MS spectrum of xylo-oligosaccharide standards; xylo-tetraose $\left(\mathrm{X}_{4}\right)$, xylo-pentaose $\left(\mathrm{X}_{5}\right)$ and xylo-hexaose $\left(\mathrm{X}_{6}\right)$. Their original molecular weights are, respectively, 546.5, 678.6 and 810.7, while their corresponding mass peaks in Fig. 1 are positioned at $m / z=569.3,701.4$ and 833.4. Taking it into consideration that oligosaccharides would be in a form of sodium $(\mathrm{m} / z=$ 23.0) cationized species $[\mathrm{M}+\mathrm{Na}]^{+}[13]$, these oligosaccharides must be detected as sodium-adducted cations, where sodium was derived from sodium trifluoroacetate. Therefore, $\mathrm{X}_{4}, \mathrm{X}_{5}$ and $\mathrm{X}_{6}$ were, respectively, detected at $m / z=569.3(\sim 546.5+23.0), 701.4(\sim 678.6+23.0)$ and $833.4(\sim 810.7+$ 23.0). Furthermore, the interval in molecular weight between these oligosaccharides was $\mathrm{m} / \mathrm{z}=132$, which corresponds to that of xylose unit $(m / z=132)$. It means that MALDI-TOF/MS analysis can detect xylo-oligosaccharides with the interval of their monomeric unit in a form of sodium-adducted cations.

MALDI-TOF/MS analyses of $O$-acetyl-4- $O$-methylglucuronoxylan as treated by hot-compressed water 
A major part of hardwood hemicellulose is known to be $O$-acetyl-4- $O$-methylglucuronoxylan [3].

MALDI-TOF/MS spectrum of hydrolyzates derived from xylan in Japanese beech as treated by semi-flow hot-compressed water at $220{ }^{\circ} \mathrm{C} / 10 \mathrm{MPa}$ suggests that it is also $O$-acetyl-4-O-methylglucuronoxylan as shown in Fig. 2. It seems quite apparent that $\beta$-1,4-glycosidic linkages of xylan in Japanese beech cleave to some extent to form various kinds of xylo-oligosaccharides. In Fig. 2, mass peaks are consecutively and regularly positioned with intervals of $m / z=42,190$ and 132 .

The $O$-acetyl-4- $O$-methylglucuronoxylan is based on a linear backbone consisting of xyloses polymerized by $\beta-1,4$-glycosidic linkage, and some of the xylose units $(\mathrm{m} / \mathrm{z}=132)$ are natively acetylated in the hydroxyl groups of C-2 and/or C-3 [3]. Since a hydrogen atom $(\mathrm{H} ; \mathrm{m} / \mathrm{z}=1)$ is in nature to be replaced with acetyl group $\left(\mathrm{CH}_{3} \mathrm{CO} ; \mathrm{m} / z=43\right)$ in acetylation of hydroxyl group $(\mathrm{OH})$, the additional molecular weight is $\Delta m / z=43-1=42$. Therefore, a molecular interval between peaks by $m / z=42$ must be attributed to acetyl group. Given that $4-O$-methylglucuronic acid $(\mathrm{m} / z=208)$ residue is attached to xylose unit by $\alpha-1,2$-glycosidic linkage [3], additional molecular weight by its residue can be obtained by subtracting molecular weights of $\mathrm{H}(\mathrm{m} / \mathrm{z}=1)$ and $\mathrm{OH}(\mathrm{m} / \mathrm{z}=17)$ to be $\Delta m / z=208-(1+17)=190$.

Taking it into consideration that the xylo-oligosaccharides have provided sodium cationized species, it can be depicted that a peak at 1761.9 is derived from xylo-oligosaccharides with $\mathrm{m} / \mathrm{z}=$ 1738.9 (= 1761.9 - 23) as $O$-acetyl-4- $O$-methylglucuronoxylan treated by semi-flow hot-compressed 
water at $220{ }^{\circ} \mathrm{C} / 10 \mathrm{MPa}$. This value corresponds to xylo-oligosaccharide which has 10 xylose units $\left(\mathrm{X}_{10}\right)$ associated with 5 acetyl groups $\left(\mathrm{Ac}_{5}\right)$ and one 4-O-methylglucuronic acid residue $\left(\mathrm{MG}_{1}\right)$ shown by $\mathrm{X}_{10} \mathrm{Ac}_{5} \mathrm{MG}_{1}[\mathrm{~m} / \mathrm{z}=1738=(132 \times 10+18)+42 \times 5+190]$. In the same way as shown in Fig. 2 , peaks at $1704.0,1572.0$ and 1530.1 can correspond to those of sodium-adducted $\mathrm{X}_{11} \mathrm{Ac}_{5}[\mathrm{~m} / z=1703$ $=(132 \times 11+18)+42 \times 5+23], \mathrm{X}_{10} \mathrm{Ac}_{5}[\mathrm{~m} / \mathrm{z}=1571=(132 \times 10+18)+42 \times 5+23]$, and $\mathrm{X}_{10} \mathrm{Ac}_{4}$ $[m / z=1529=(132 \times 10+18)+42 \times 4+23]$, respectively.

Fig. 3 shows the temperature profile for the hot-compressed water treatment of Japanese beech and the obtained spectra from different hydrolyzate fragments at different temperatures under 10 MPa. Fig. 3a shows a distinctive MALDI-TOF/MS spectrum of the obtained hydrolyzate fractions at a temperature range from $150{ }^{\circ} \mathrm{C}$ to $210{ }^{\circ} \mathrm{C}$ under $10 \mathrm{MPa}$. Although this spectrum was analyzed thoroughly, no characteristic peaks were observed. Therefore, it is implied that $O$-acetyl-4- $O$-methylglucuronoxylan cannot be decomposed under a given condition. In fact, the previous study by HPLC analyses [10] showed that xylo-oligosaccharides derived from xylan in Japanese beech were not detected in a temperature range between $150{ }^{\circ} \mathrm{C}$ and $210{ }^{\circ} \mathrm{C}$ under $10 \mathrm{MPa}$.

Fig. $3 b$ is the MALDI-TOF/MS spectrum of the hydrolyzate derived from Japanese beech obtained at $210{ }^{\circ} \mathrm{C} / 10 \mathrm{MPa}$, where consecutive peaks marked with filled circle $\left(\mathrm{X}_{n} \mathrm{Ac} \mathrm{c}_{m}\right)$ and with filled triangle $\left(\mathrm{X}_{n} \mathrm{Ac}_{m} \mathrm{MG}_{i}\right)$ are shown. At this condition, these peaks were firstly observed, which indicates that the xylan began to be hydrolyzed into $O$-acetyl-4- $O$-methylglucuronoxylo-oligosaccharides and 
$O$-acetyl-xylo-oligosaccharides $\left(\mathrm{X}_{n} \mathrm{Ac}_{m}\right)$ at $210{ }^{\circ} \mathrm{C} / 10 \mathrm{MPa}$. In Fig. 3b, the degree of polymerization (DP) of the $\mathrm{X}_{n} \mathrm{Ac}_{m}$, defined as the number $(n)$ of polymerized units, is from $11\left(\mathrm{X}_{11} \mathrm{Ac}_{4}\right)$ to 13 $\left(\mathrm{X}_{13} \mathrm{Ac}_{5}\right)$, while the DP of $\mathrm{X}_{n} \mathrm{Ac}_{m} \mathrm{MG}_{i}$ detected is $9\left(\mathrm{X}_{9} \mathrm{Ac}_{5} \mathrm{MG}_{1}\right.$ and $\left.\mathrm{X}_{9} \mathrm{Ac}_{7} \mathrm{MG}_{1}\right)$. The number of acetyl groups per a xylose unit in these $\mathrm{X}_{n} \mathrm{Ac}_{m} \mathrm{MG}_{i}$ was from $0.56(=5 / 9)$ to 0.78 (=7/9). According to Timell [3], 6.8 acetyl groups and 1.6 glucuronic acid residues per 10 xylose units were found in xylan of American beech (Fagus grandifolia). Therefore, the oligosaccharides obtained in hot-compressed water treatment could reflect the structure of the native xylan in Japanese beech.

Fig. 3c shows the typical MALDI-TOF/MS spectrum of the hydrolyzate obtained at a temperature range from $220{ }^{\circ} \mathrm{C}$ to $230{ }^{\circ} \mathrm{C}$ under $10 \mathrm{MPa}$. In this spectrum, mass peaks marked with filled circle $\left(\mathrm{X}_{n} \mathrm{Ac}_{m}\right)$ and with filled triangle $\left(\mathrm{X}_{n} \mathrm{Ac}_{m} \mathrm{MG}_{i}\right)$ were simultaneously observed. It reveals that $O$-acetyl-4-O-methylglucuronoxylo-oligosaccharides $\left(\mathrm{X}_{n} \mathrm{Ac}_{m} \mathrm{MG}_{i}\right)$ and $O$-acetyl-xylo-oligosaccharides $\left(\mathrm{X}_{n} \mathrm{Ac}_{m}\right)$ were recovered. In a similar manner in Fig. $3 \mathrm{~b}$, the DP of the $\mathrm{X}_{n} \mathrm{Ac}_{m}$ observed as peaks was in a range between 10 and 13, while that of the $O$-acetyl-4-O-methylglucuronoxylo-oligosaccharides $\left(\mathrm{X}_{n} \mathrm{Ac}_{m} \mathrm{MG}_{i}\right)$ between 9 and 11 . The results were similar to those in Fig. $3 b$.

Fig. $3 \mathrm{~d}$ is the mass spectrum of the hydrolyzate obtained immediately after the treatment temperature reached to $230{ }^{\circ} \mathrm{C}$ under $10 \mathrm{MPa}$. Consecutive peaks marked with filled circle $\left(\mathrm{X}_{n} \mathrm{Ac}_{m}\right)$ are observed as well, whereas number of those peaks marked with filled triangle $\left(\mathrm{X}_{n} \mathrm{Ac}_{m} \mathrm{MG}_{i}\right)$ in Fig. $3 \mathrm{~d}$ is less than in Fig. 3c. It implies that 4-O-methylglucuronic acid residue had been hydrolyzed at 
the temperature range from $220{ }^{\circ} \mathrm{C}$ to $230{ }^{\circ} \mathrm{C}$ under $10 \mathrm{MPa}$. It is shown that the $O$-acetyl-xylo-oligosaccharides $\left(\mathrm{X}_{n} \mathrm{Ac}_{m}\right)$ were observed up to tridecamer ( $\mathrm{DP}=13$ ), which is similar results in Fig $3 b$ and c.

Fig. 3e represents the mass spectrum of the hydrolyzate obtained at $230{ }^{\circ} \mathrm{C} / 10 \mathrm{MPa}$ in its early stage. Although mass peaks marked with filled circle $\left(\mathrm{X}_{n} \mathrm{Ac}_{m}\right)$ were shown in this spectrum, peaks with filled triangle $\left(\mathrm{X}_{n} \mathrm{Ac}_{m} \mathrm{MG}_{i}\right)$ were no longer observed. This means that all the xylo-oligosaccharides recovered had some acetyl groups but not 4- $O$-methylglucuronic acid residues. Therefore, it seems apparent that 4-O-methylglucuronic acid residues are hydrolyzed between $220{ }^{\circ} \mathrm{C}$ and $230{ }^{\circ} \mathrm{C} / 10 \mathrm{MPa}$. In addition, the evidence from MALDI-TOF/MS analysis was in agreement with that from the previous HPLC analysis, where 4-O-methylglucuronic acid residue was detected in the soluble portion [10]. As in the previous spectra, the maximum DP of the xylo-oligosaccharides detected was 13.

On the other hand, peaks marked with empty square are simultaneously observed together with peaks of the xylo-oligosaccharides marked as the filled circle. These peaks appears in Fig. 3e as well and were positioned with an interval of $\mathrm{m} / \mathrm{z}=162$, which corresponds to the molecular weight of anhydrohexose $(=180-18=162)$. At $230{ }^{\circ} \mathrm{C} / 10 \mathrm{MPa} / 15 \mathrm{~min}$, para-crystalline cellulose was hydrolyzed $[10,11]$. Thus, it is implied that peaks marked with empty squares in Figs. $3 \mathrm{e}$ and $3 \mathrm{f}$ were derived from cellulose hydrolyzates, i.e., cello-oligosaccharides.

In the spectrum of Fig. 3f, although there exist less peaks than in Fig. 3e, xylo-oligosaccharides up 
to tridecamer $(\mathrm{DP}=13)$ attached by acetyl groups are observed. It indicates that acetyl groups are more difficult to be hydrolyzed than 4-O-methylglucuronic acid residue at a given condition.

In Fig. 4, the lower mass range of the corresponding spectra shown in Fig. 3 are depicted. Due to no consecutive peaks derived from xylo-oligosaccharides, Fig. 4a indicates that the decomposition of xylan in Japanese beech did not occur at $150-210{ }^{\circ} \mathrm{C} / 10 \mathrm{MPa}$.

Fig. $4 \mathrm{~b}$ is the spectrum of hydrolyzates derived from Japanese beech obtained at $210{ }^{\circ} \mathrm{C} / 10 \mathrm{MPa}$., while the characteristic peaks are not seen. Since HPLC analyses in the previous study [10] reported that xylo-oligosaccharides up to hexamer began to be recovered at $210{ }^{\circ} \mathrm{C} / 10 \mathrm{MPa}$, the xylan decomposition might not have progressed enough to give such xylo-oligosaccharides which could not be detected in the lower mass range in MALDI-TOF/MS spectrum.

On the other hand, MALDI-TOF/MS spectrum in Fig. 4c has the mass peaks of the obtained hydrolyzed xylan at $220{ }^{\circ} \mathrm{C} / 10 \mathrm{MPa}$, where the mass peaks are observed at $\mathrm{m} / z=743.9$ and 785.9 . They correspond to the molecular weight of the sodium-adducted $\mathrm{X}_{5} \mathrm{Ac}_{1}[\mathrm{~m} / z=743=(132 \times 5+18)$ $+42 \times 1+23]$ and $\mathrm{X}_{5} \mathrm{Ac}_{2}[\mathrm{~m} / \mathrm{z}=785=(132 \times 5+18)+42 \times 2+23]$, respectively.

However, in Fig. 4d, an additional mass peak appears at $m / z=701.9$. The value corresponds to the molecular weight of the sodium-adducted $\mathrm{X}_{5}[\mathrm{~m} / \mathrm{z}=701=(132 \times 5+18)+23]$. Additionally, the sodium-adducted $\mathrm{X}_{5} \mathrm{Ac}_{1}$ and $\mathrm{X}_{5} \mathrm{Ac}_{2}$ were detected as well. Similarly, Figs. $4 \mathrm{e}$ and $\mathrm{f}$ also show the same peaks. Therefore, at $230{ }^{\circ} \mathrm{C} / 10 \mathrm{MPa}$, only a few of acetyl groups were hydrolyzed.

These results indicate that xylo-polysaccharides have released acetyl groups to be some 
oligosaccharides without any acetyl groups during hot-compressed water treatment at $230{ }^{\circ} \mathrm{C} / 10$ $\mathrm{MPa}$ and/or that some portions of xylo-polysaccharides with which acetyl groups are not attached were hydrolyzed to be xylo-oligosaccharides. Consequently, xylo-oligosacchrides without any acetyl groups were resulted.

Hydrolysis pathway of $O$-acetyl-4- $O$-methylglucuronoxylan

Based on these lines of evidence, the hydrolysis pathway of $O$-acetyl-4- $O$-methylglucuronoxylan was proposed. Fig. 5 shows the obtained pathway of native xylan as treated by hot-compressed water. Under $10 \mathrm{MPa}$, the xylan started to be hydrolyzed from $210{ }^{\circ} \mathrm{C}$. By $220{ }^{\circ} \mathrm{C}$, $O$-acetyl-4- $O$-methylglucuronoxylo-oligosaccharides were first recovered as hydrolyzates. In a vicinity of the treatment temperature around $230{ }^{\circ} \mathrm{C}$ or lower, $4-O$-methylglucuronic acid residue was, then, completely hydrolyzed. On the other hand, acetyl groups remained unchanged with xylo-oligosaccharides. Some of the oligosaccharides were also further hydrolyzed to be lower molecular weight compounds such as xylo-oligosaccharides without any residues, acetic acid and xylose. Additionally, Fig. 5 suggests that decomposition products can trace back to the original $O$-acetyl-4-O-methylglucuronoxylan in wood cell wall through the hydrolysis pathway. The first oligosaccharides were more or less to be $O$-acetyl-4- $O$-methylglucuronoxylo-oligosaccharide with nine xylose units associated with seven acetyl groups and one 4-O-methylglucuronic acid residue 
$\left(\mathrm{X}_{9} \mathrm{Ac}_{7} \mathrm{MG}_{1}\right)$. This structure seems to be very likely to be the molecular structures by the natural $O$-acetyl-4- $O$-methylglucuronoxylan by only a cleavage of $\beta$-1,4-glycosidic linkage in xylan backbone. Thus, depending upon the temperature and pressure of water, xylo-oligosaccharides which preserve the original structure of $O$-acetyl-4- $O$-methylglucuronoxylan in wood could be obtained with semi-flow hot-compressed water treatment. These results indicate that MALDI-TOF/MS analysis would be a powerful tool for speculating the native structure as well as analyzing xylan-derived products as treated by semi-flow hot-compressed water. 


\section{Conclusions}

Japanese beech hemicellulose, which is mainly dominated with $O$-acetyl-4-O-methylglucuronoxylan

[3], was hydrolyzed with semi-flow hot-compressed water treatment, and the obtained hydrolyzates were then studied by MALDI-TOF/MS analyses. The obtained result of MALDI-TOF/MS analyses indicated that the hydrolysis of xylan in Japanese beech started at $210{ }^{\circ} \mathrm{C}$ under $10 \mathrm{MPa}$ by preferential cleavage of $\beta$-1,4-glycosidic linkage of xylan backbone. The resulting xylo-oligosaccharides at $210-220{ }^{\circ} \mathrm{C} / 10 \mathrm{MPa}$ were found out to have original acetyl groups, remained unchanged, attached to them. Additionally, some of the oligosaccharides contained one 4- $O$-methylglucuronic acid residue. However, xylo-oligosaccharides recovered at $230{ }^{\circ} \mathrm{C} / 10 \mathrm{MPa}$ had no 4-O-methylglucuronic acid residues, while acetyl groups remained attached to xylo-oligosaccharides. Therefore, 4- $O$-methylglucuronic acid residue must be hydrolyzed and desorbed from xylan in Japanese beech backbone, prior to the deacetylation. Thus, these lines of information lead to the hydrothermal hydrolysis pathway as shown in Fig. 5, where $\beta$-1,4-glycosidic linkages in the xylan backbone were first hydrolyzed, and then, $\alpha-1,2$-glycosidic linkage and finally ester linkage between acetyl group and xylose unit, in hot-compressed water treatment at a range of temperature from $210-230{ }^{\circ} \mathrm{C}$ under $10 \mathrm{MPa}$.

According to Timell's data [3], in American beech (Fagus grandifolia), 6.8 acetyl groups and 1.6 glucuronic acid residues out of 10 xylose units are contained. On the other hand, in Fig. 3b, xylo-oligosaccharides derived from the xylan in Japanese beech as treated with hot-compressed 
water contained about 7.8 acetyl groups and 1.0 4-O-methylglucuronic acid residue out of 10 xylose units. Although the number of acetyl groups was slightly higher, it must be a native structure due to an intrinsic difference between Japanese beech and hardwoods in North America studied by Timell [3], because the present study clearly presented the evidence that acetyl groups show a resistance against deacetylation prior to removal of 4-O-methylglucuronic acid residue. Therefore, the obtained result must be in good agreement with Timell's work $[3,16]$.

Since the semi-flow hot-compressed water treatment utilizes only water as a reagent, obtained results will lead to isolation of the genuinely native xylan and thus further elucidation of its structure including accurate positions, steric configuration, and tacticity of acetyl groups and 4- $O$-methylglucuronic acid residues in xylan backbone, which will eventually provide useful information for effective utilization of xylan derivatives and the related chemicals in the future. 


\section{Acknowledgements}

This work has been done as part of the JST (ALCA) project "Ethanol Production with

Acetic Acid Fermentation from Lignocellulosics." The authors gratefully acknowledge all the support. 


\section{References}

1. Core HA, Coté WA, Day AC (1979) Wood structure and identification. Syracuse University Press, Syracuse.

2. Timell TE (1957) Carbohydrate composition of ten North American species of wood. Tappi, 40:568-572

3. Timell TE (1964) Wood hemicelluloses: Part I., Adv Carbohyd Chem 19:247-302

4. Timell TE (1964) Wood hemicelluloses: Part II. Adv Carbohyd Chem 20:409-483

5. Timell TE (1960) Isolation and properties of an $O$-acetyl-4- $O$-methylglucurono-xyloglycan from the wood of white birch (Betula papyrifera) 1. J Am Chem Soc 82:5211-5215

6. Rosell KG, Svensson S (1975) Studies of the distribution of the 4-O-methyl-d-glucuronic acid residues in birch xylan. Carbohyd Res 42:297-304

7. Caregg PJ (1962) Partial acetylation studies on benzyl 4-O-methyl-3-D-xylopyranoside. Acta Chem Scand 16:1849-1857

8. Reicher F, Corrêa JB, Gorin PA (1984) Location of $O$-acetyl groups in the acidic d-xylan of mimosa scabrella (bracatinga). A study of $O$-acetyl group migration. Carbohydr Res $135: 129-140$

9. Reicher F, Gorin PA, Sierakowski MR, Correa JBC (1989) Highly uneven distribution of $O$-acetyl groups in the acidic D-xylan of Mimosa scabrella bracatinga). Cabohydr Res $193: 23-31$ 
10. Lu X, Yamauchi K, Phaiboonsilpa N, Saka S (2009) Two-step hydrolysis of Japanese beech as treated by semi-flow hot-compressed water. J Wood Sci, 55:367-375

11. Phaiboonsilpa N, Yamauchi K, Lu X, Saka S (2010) Two-step hydrolysis of Japanese cedar as treated by semi-flow hot-compressed water. J Wood Sci 56:331-338

12. Kruse A, Dinjus E (2007) Hot compressed water as reaction medium and reactant: Properties and synthesis reactions. J Supercrit Fluids 39:362-380

13. Mele A, Malpezzi L (2000) Noncovalent association phenomena of 2, 5-dihydroxybenzoic acid with cyclic and linear oligosaccharides. A matrix-assisted laser desorption/ionization time-of-flight mass spectrometric and X-ray crystallographic study. J Am So Mass Spectrom 11:228-236

14. Gross JH (2004) Matrix-Assisted Laser Desorption/Ionization. In: Mass spectrometry - A Textbook. Springer-Verlag, Berlin pp 420-421

15. Ehara K, Saka S (2002) A comparative study on chemical conversion of cellulose between the batch-type and flow-type systems in supercritical water. Cellulose 9:301-311

16. Timell TE (1967) Recent progress in the chemistry of wood hemicelluloses. Wood Sci Technol 1:45-70 


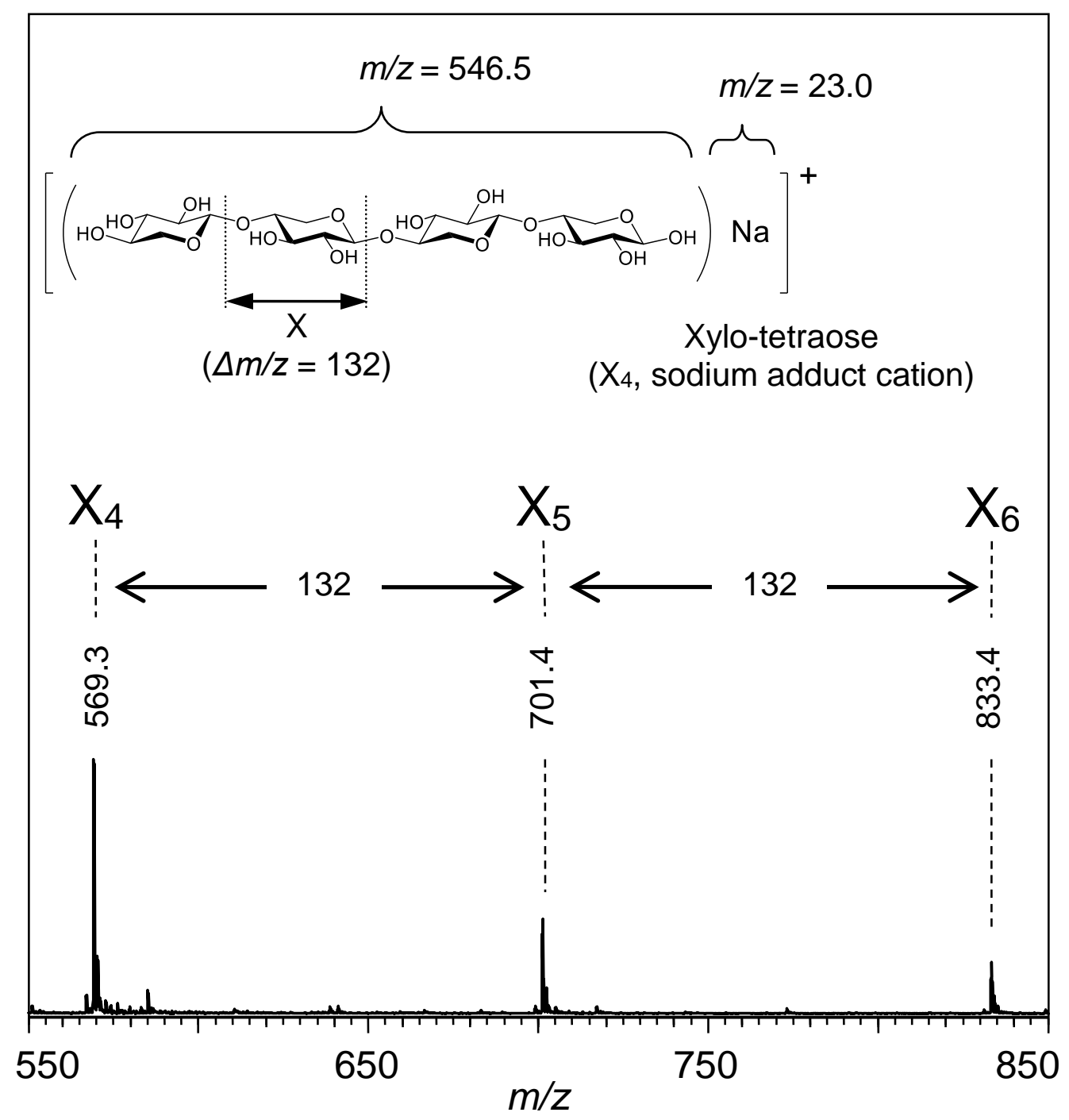

Fig. 1 MALDI-TOF/MS spectrum of xylo-tetraose $\left(\mathrm{X}_{4}\right)$, xylo-pentaose $\left(\mathrm{X}_{5}\right)$ and xylo-hexaose $\left(\mathrm{X}_{6}\right)$ as xylo-oligosaccharide standards. All the peaks shown are of sodium adduct cations. 


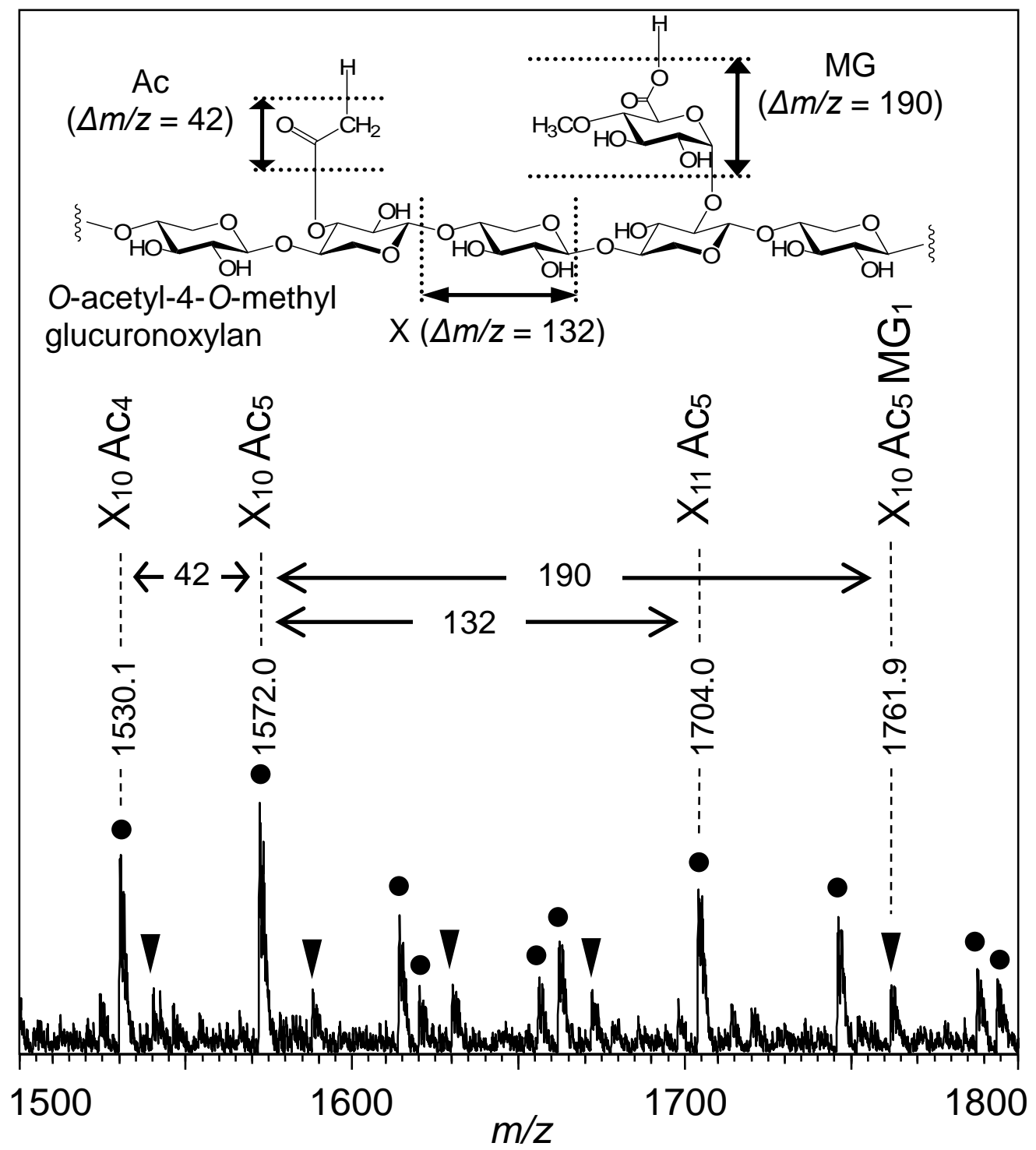

Fig. 2 MALDI-TOF/MS spectrum of hydrolyzates derived from Japanese beech as treated by semi-flow hot-compressed water at $220{ }^{\circ} \mathrm{C} / 10 \mathrm{MPa}$. The filled circle $(\bullet)$ indicates xylo-oligosaccharide $\left(\mathrm{X}_{n}, n=1,2, \ldots\right)$ associated with some acetyl groups (Ac), while the filled triangle ( $\mathbf{V})$ stands for xylo-oligosaccharide with one 4-O-methylglucuronic acid residue (MG) as well as some acetyl groups. All the peaks shown are of sodium adduct cations. 


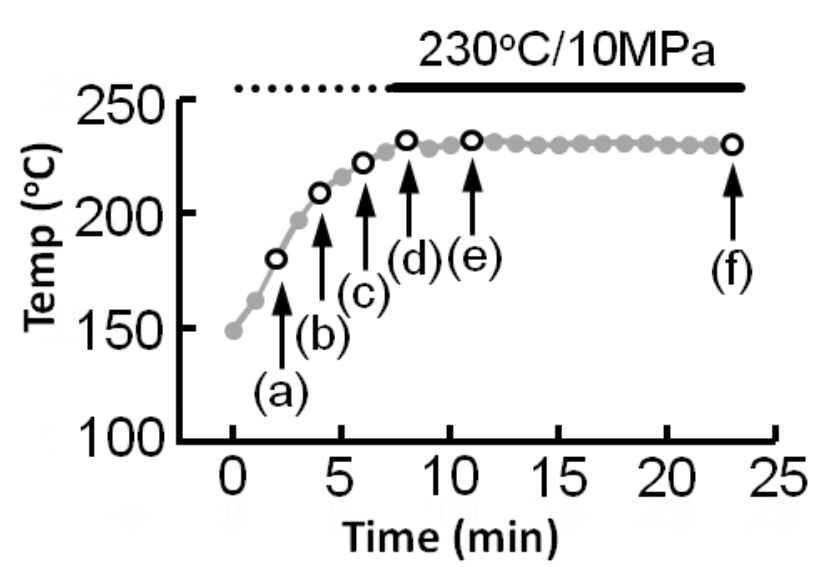

Fig. 3 The temperature profile for the hot-compressed water treatment of Japanese beech and MALDI-TOF/MS spectra of the obtained hydrolyzate fractions of hydrolyzed xylan at $180{ }^{\circ} \mathrm{C}$ (a), $210{ }^{\circ} \mathrm{C} \mathrm{(b),} 220{ }^{\circ} \mathrm{C}$ (c) and $230{ }^{\circ} \mathrm{C}(\mathrm{d}),(\mathrm{e})$ and (f) under 10 $\mathrm{MPa}$. All the peaks shown are of sodium adduct cations. $\mathrm{X}$ : xylose unit, $n$ : number of xylose units, Ac: acetyl group, $m$ : number of acetyl groups, MG: 4-O-methylglucuronic residue, $i$ : number 4-O-methylglucuronic residue. acid of acid

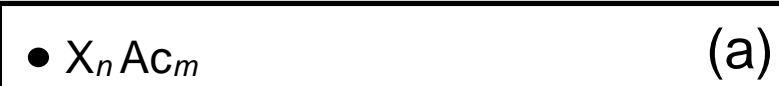

V $\mathrm{X}_{n} \mathrm{Ac}_{m} \mathrm{MG}_{i}$

$\square$ Cello-oligosaccharide

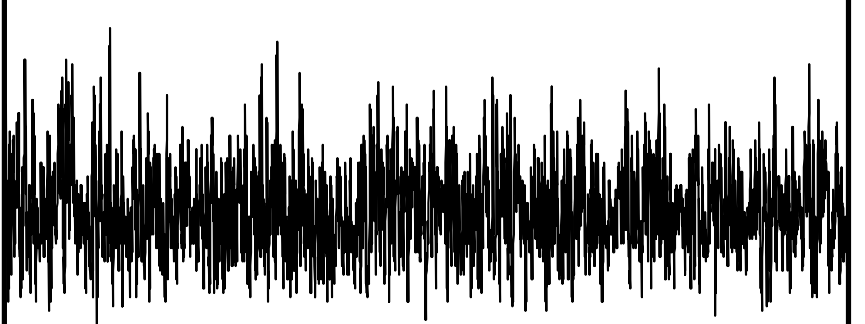

(b)
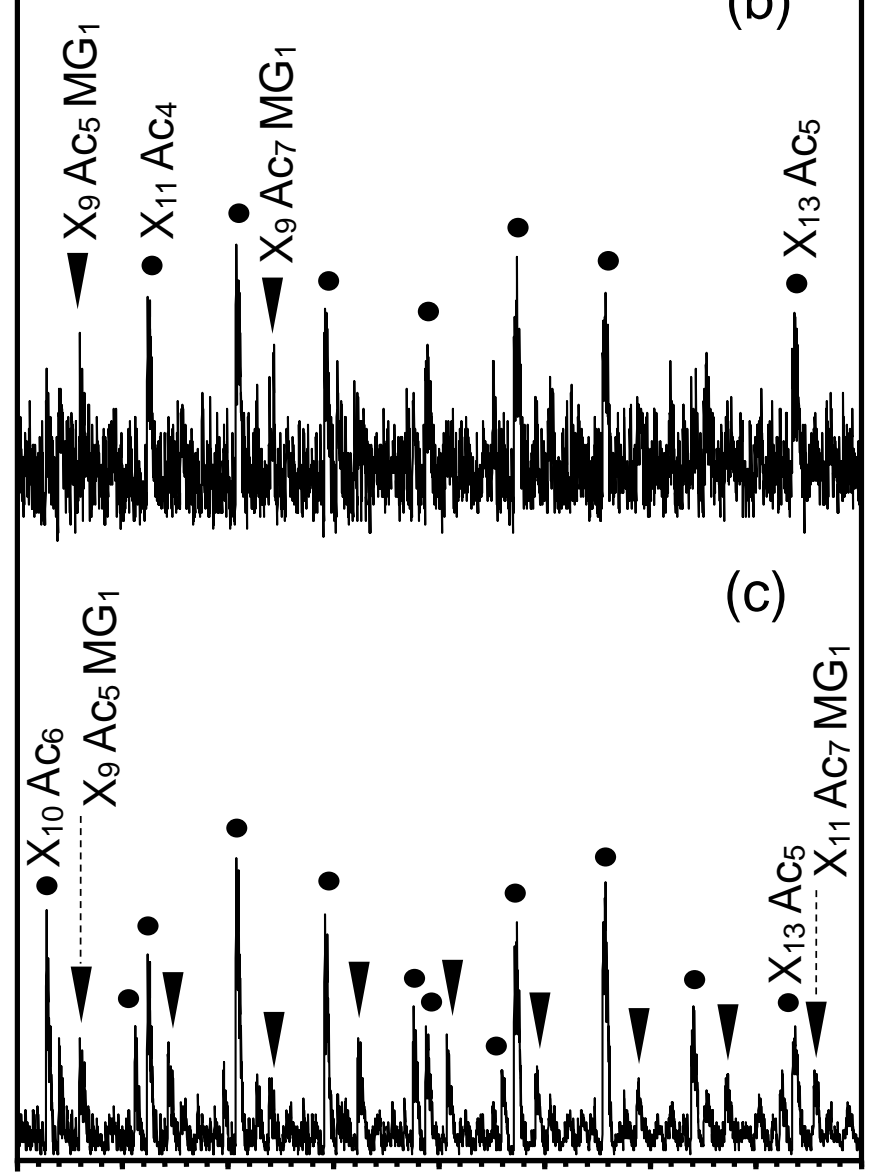

1600 


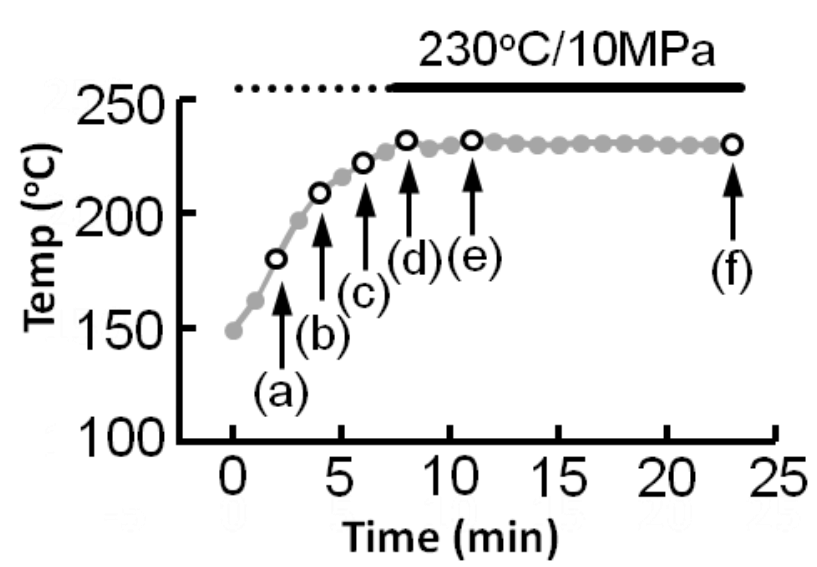

Fig. 4 The temperature profile for the hot-compressed water treatment of Japanese beech and MALDI-TOF/MS spectra of xylo-pentaose $\left(\mathrm{X}_{5}\right)$ as xylooligosaccharide standard (A) and the obtained hydrolyzate fractions of hydrolyzed xylan at $180{ }^{\circ} \mathrm{C}$ (a), $210{ }^{\circ} \mathrm{C} \quad$ (b), $220{ }^{\circ} \mathrm{C}$ (c) and $230{ }^{\circ} \mathrm{C}(\mathrm{d})$, (e) and (f) under $10 \mathrm{MPa}$. All the peaks shown are of sodium adduct cations. The empty circle represents xylo- pentaose with no acetyl group, while the filled circle stands

$O$-acetyl-xylo-pentaoses $\left[\mathrm{X}_{5} \mathrm{Ac}_{1}\right.$ : $\left.m / z=743, \mathrm{X}_{5} \mathrm{Ac}_{2}: m / z=785\right]$.
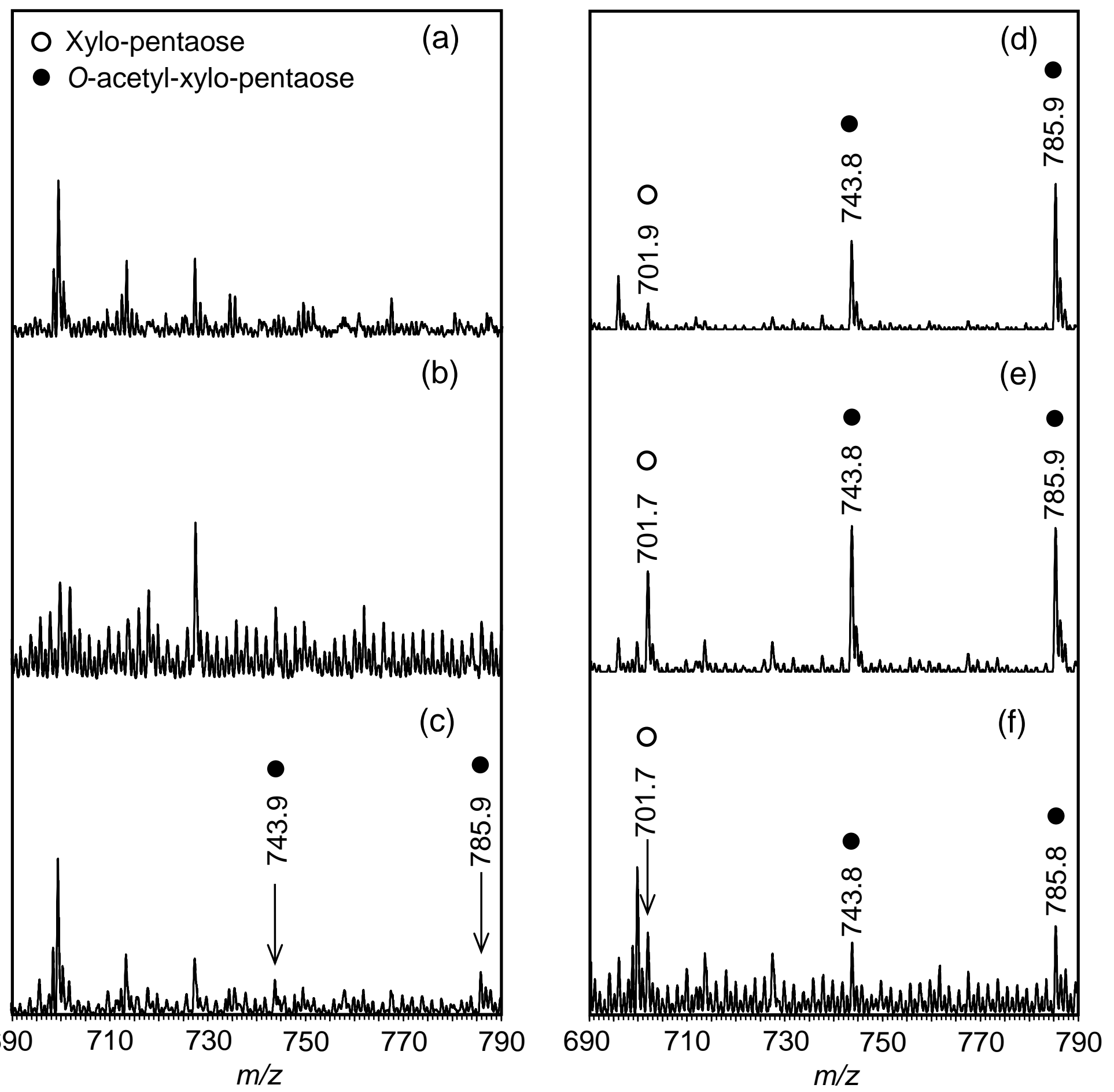


\section{O-acetyl-4-O-methylglucuronoxylan (Native)}

$$
210-220{ }^{\circ} \mathrm{C} / 10 \mathrm{MPa}
$$

O-acetyl-4-O-methylglucuronoxylo-oligosaccharides $\left(\mathrm{X}_{n} \mathrm{Ac}_{m} \mathrm{MG}_{i}\right)$
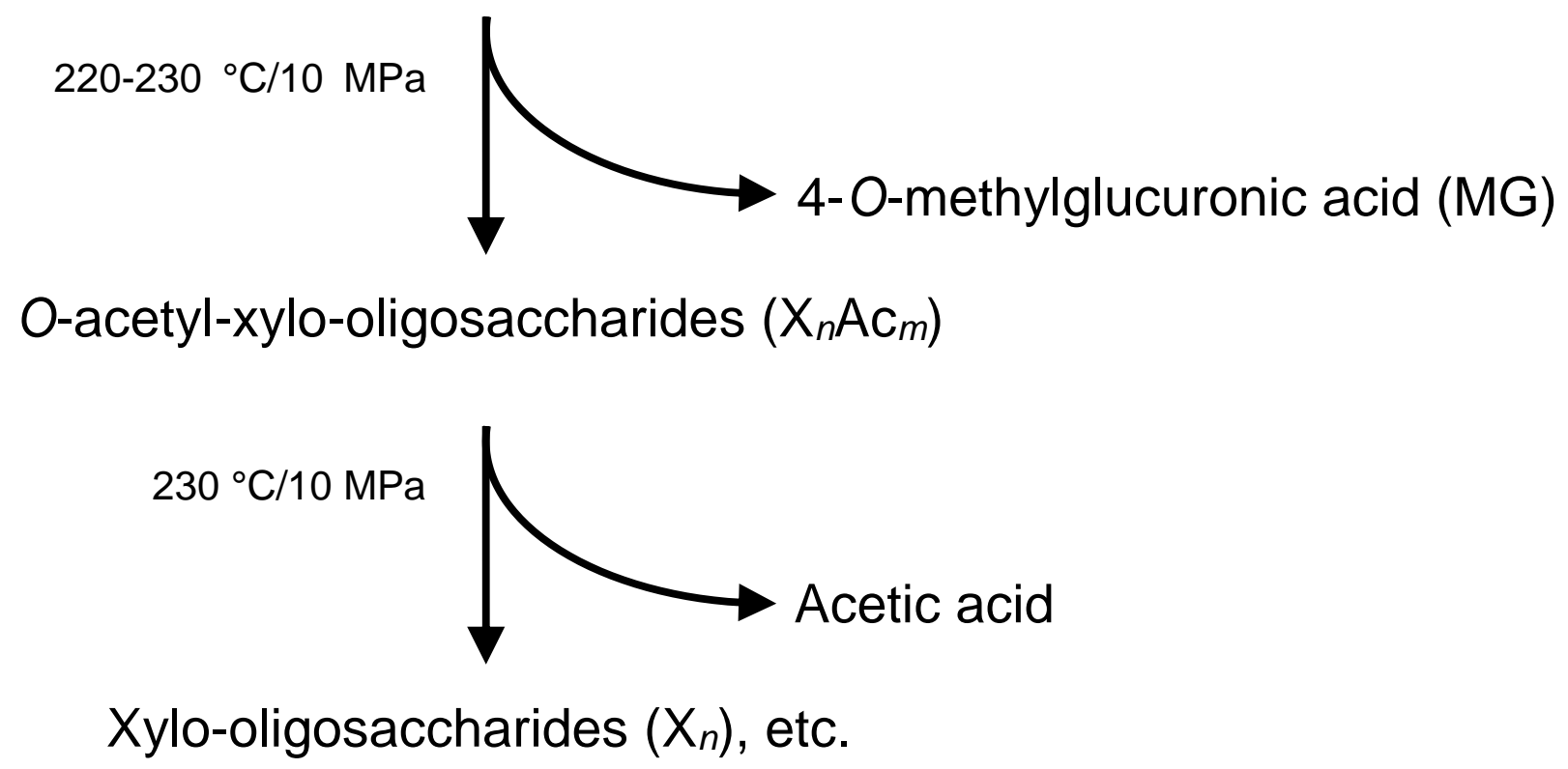

Fig. 5 Hydrolysis pathway of native $O$-acetyl-4- $O$-methylglucuronoxylan as treated by hot-compressed water. 